\title{
On the Uniform Convergence of Gaussian Quadrature Rules for Cauchy Principal Value Integrals and Their Derivatives
}

\author{
By N. I. Ioakimidis
}

\begin{abstract}
The convergence of the aforementioned quadrature rules for integrands possessing Hölder-continuous derivatives of an appropriate order is proved to be uniform and not only pointwise. The rate of convergence is also established and an application to the numerical solution of singular integral equations is made.
\end{abstract}

1. Introduction. In a recent short communication [8] we considered the convergence of Gaussian quadrature rules for the numerical evaluation of derivatives of Cauchy principal value integrals of the form

$$
\begin{aligned}
I^{(p)}(x) & =\frac{d^{p}}{d x^{p}} f_{a}^{b} w(t) \frac{f(t)}{t-x} d t \\
& =p ! f_{a}^{b} w(t) \frac{f(t)}{(t-x)^{p+1}} d t, \quad a<x<b, p=1,2, \ldots
\end{aligned}
$$

(For $p=0, I^{(0)}(x)$ denotes simply an ordinary Cauchy principal value integral.) The last integral in (1) should be interpreted as a finite-part integral [15]. Yet, instead of using the complicated definition of such an integral proposed by Hadamard, we prefer to define it here, equivalently, by (1) as a derivative of a Cauchy principal value integral. This is a common practice.

For the construction of the aforementioned rules, we rewrite (1) as

$$
\begin{aligned}
I^{(p)}(x)= & p ! \int_{a}^{b} \frac{w(t)}{(t-x)^{p+1}}\left[f(t)-\sum_{k=0}^{p} \frac{f^{(k)}(x)}{k !}(t-x)^{k}\right] d t \\
& +p ! \sum_{k=0}^{p} \frac{f^{(k)}(x)}{k !} f_{a}^{b} \frac{w(t)}{(t-x)^{p-k+1}} d t, \quad a<x<b
\end{aligned}
$$

assuming that $f \in C^{p+1}[a, b]$ and that $w(t)$ is a nonnegative generalized Höldercontinuous function on $[a, b]$ (that is, a function which is Hölder-continuous on every closed subinterval of $[a, b]$ not containing the endpoints $a$ and $b$ and near these endpoints may have integrable algebraic or algebraicologarithmic singularities

Received February 8, 1983; revised July 15, 1983, January 17, 1984 and May 30, 1984.

1980 Mathematics Subject Classification. Primary 65D30, 65D32; Secondary 41A55, 65R20. 
[17]). Then the first integral in (2) can be evaluated by the classical Gaussian quadrature rule [1]

$$
\int_{a}^{b} w(t) h(t) d t=\sum_{i=1}^{n} \mu_{i, n} h\left(t_{i, n}\right) d t+E_{n}(f)
$$

for the weight function $w(t)$ on $[a, b]$ (where $t_{i, n}$ are the nodes, $\mu_{i, n}$ the weights, $\mu_{i, n}>0$, and $E_{n}$ the error term) and the resulting quadrature rule for $I^{(p)}(x)$ is called the Gaussian quadrature rule for $I^{(p)}(x)$. By defining the polynomial

$$
p_{n}(x)=\prod_{i=1}^{n}\left(x-t_{i, n}\right)
$$

and the corresponding function of the second kind

$$
q_{n}(x)=f_{a}^{b} w(t) \frac{p_{n}(t)}{t-x} d t, \quad a<x<b,
$$

(where, for the sake of generality, we have used the symbol $f$ instead of $f$ ), we have for the integrals in the sum in the right side of (2)

$$
f_{a}^{b} \frac{w(t)}{(t-x)^{p-k+1}} d t=\frac{1}{(p-k) !} q_{0}^{(p-k)}(x), \quad a<x<b .
$$

We can also consider the cases where $x=a$ or $x=b$. In these cases, we define $I^{(p)}(x)$ directly from (2), defining the last integrals in it (which are not regular integrals) in some appropriate sense (e.g., the finite-part sense [15]) or, more generally, as

$$
f_{a}^{b} \frac{w(t)}{(t-a)^{m}} d t=A_{m}, \quad f_{a}^{b} \frac{w(t)}{(t-b)^{m}} d t=B_{m}, \quad m=1,2, \ldots,
$$

where $A_{m}$ and $B_{m}$ are appropriately or arbitrarily selected constants. Then (taking into account (5)), we rewrite (6a) equivalently by defining the quantities $q_{0}^{(p-k)}(a)$ and $q_{0}^{(p-k)}(b)$ by

$$
q_{0}^{(p-k)}(a)=(p-k) ! A_{p-k+1}, \quad q_{0}^{(p-k)}(b)=(p-k) ! B_{p-k+1} .
$$

(These quantities should not be understood as $(p-k)$ th derivatives of $q_{0}(x)$ at $x=a, b ; q_{0}(x)$ is generally not differentiable at these points.) In this way, (5) holds true also for $x=a, b$. The same happens for (2), which we take as the definition of $I^{(p)}(x)$ in (1) for these special values of $x$. As will become clear below, the values of $A_{m}$ and $B_{m}$ have no influence on the quadrature error and the convergence results, simply because just the first integral in the right side of (2) is approximated by using the quadrature rule (3); but this integral is simply a regular integral. Finally, again for $x=a, b, q_{n}^{(p)}(x)$ are defined by (2) with $f(t) \equiv p_{n}(t)$ and (6a) taken into consideration. (Of course, (4b) also holds true for $x=a, b$.)

Now, by using (3) and (5), we obtain from (2) the following explicit form for the Gaussian quadrature rule for $I^{(p)}(x)$ :

$$
\begin{aligned}
I^{(p)}(x)= & p ! \sum_{i=1}^{n} \frac{\mu_{i, n}}{\left(t_{i, n}-x\right)^{p+1}}\left[f\left(t_{i, n}\right)-\sum_{k=0}^{p} \frac{f^{(k)}(x)}{k !}\left(t_{i, n}-x\right)^{k}\right] \\
& +\sum_{k=0}^{p}\left(\begin{array}{l}
p \\
k
\end{array}\right) q_{0}^{(p-k)}(x) f^{(k)}(x)+E_{n}(f ; x)
\end{aligned}
$$


( $E_{n}$ denoting again the error term). Of course, for $x=t_{j, n}(j=1(1) n)$ the $j$ th term of the quadrature sum in (7) is equal to $\mu_{j, n} f^{(p+1)}\left(t_{j, n}\right) /(p+1)$. The quadrature rule (7) was also derived in [8] by a different approach. For $x \neq t_{j, n}$ an equivalent form of (7) is [8]

$$
\begin{aligned}
I^{(p)}(x)= & p ! \sum_{i=1}^{n} \frac{\mu_{i, n}}{\left(t_{i, n}-x\right)^{p+1}} f\left(t_{i, n}\right)+\sum_{k=0}^{p}\left(\begin{array}{l}
p \\
k
\end{array}\right) K_{n}^{(p-k)}(x) f^{(k)}(x) \\
& +E_{n}(f ; x), \quad K_{n}(x)=q_{n}(x) / p_{n}(x), x \neq t_{j, n}, j=1(1) n .
\end{aligned}
$$

It can also be mentioned that the corresponding forms of (7) for $x=t_{j, n}$ are complicated, but easy to derive from (7) (or even (8)) by a limiting procedure. We confine ourselves to presenting them only for $p=0$ [11] (see also [16]) and $p=1$ $[10]^{*}$

$$
\begin{aligned}
& I^{(0)}\left(t_{j, n}\right)=\sum_{\substack{i=1 \\
i \neq j}}^{n} \frac{\mu_{i, n}}{t_{i, n}-t_{j, n}} f\left(t_{i, n}\right) \\
& +\frac{1}{p_{n}^{\prime}\left(t_{j, n}\right)}\left[q_{n}^{\prime}\left(t_{j, n}\right)-\frac{1}{2} \mu_{j, n} p_{n}^{\prime \prime}\left(t_{j, n}\right)\right] f\left(t_{j, n}\right) \\
& +\mu_{j, n} f^{\prime}\left(t_{j, n}\right)+E_{n}\left(f ; t_{j, n}\right), \\
& I^{(1)}\left(t_{j, n}\right)=\sum_{\substack{i=1 \\
i \neq j}}^{n} \frac{\mu_{i, n}}{\left(t_{i, n}-t_{j, n}\right)^{2}} f\left(t_{i, n}\right)+\frac{1}{2 p_{n}^{\prime 2}\left(t_{j, n}\right)} \\
& \times\left\{\left[q_{n}\left(t_{j, n}\right)\left(\frac{p_{n}^{\prime \prime 2}\left(t_{j, n}\right)}{2 p_{n}^{\prime}\left(t_{j, n}\right)}-\frac{1}{3} p_{n}^{\prime \prime \prime}\left(t_{j, n}\right)\right)\right.\right. \\
& \left.-q_{n}^{\prime}\left(t_{j, n}\right) p_{n}^{\prime \prime}\left(t_{j, n}\right)+q_{n}^{\prime \prime}\left(t_{j, n}\right) p_{n}^{\prime}\left(t_{j, n}\right)\right] f\left(t_{j, n}\right) \\
& \left.+\left[-q_{n}\left(t_{j, n}\right) p_{n}^{\prime \prime}\left(t_{j, n}\right)+2 q_{n}^{\prime}\left(t_{j, n}\right) p_{n}^{\prime}\left(t_{j, n}\right)\right] f^{\prime}\left(t_{j, n}\right)\right\} \\
& +\frac{1}{2} \mu_{j, n} f^{\prime \prime}\left(t_{j, n}\right)+E_{n}\left(f ; t_{j, n}\right) \text {. }
\end{aligned}
$$

The quadrature rule (7) (or, almost equivalently, (8)) is a generalization of the corresponding rule for ordinary Cauchy principal value integrals $(p=0)$. The Gauss-Legendre quadrature rule for this class of integrals was suggested in [6]. The general Gaussian quadrature rule for the same class of integrals was obtained in [7], [11]. Convergence results in the same case $(p=0)$ were proved in [2], [5], [9]. The most important of these results is that by Elliott and Paget [5], who proved that in this case if $f \in C^{1}[a, b]$, then the Gaussian quadrature rule for $I^{(0)}(x)$ (a special case of (7)) converges (see also [16]). A stronger result in [22] for the special case of the Gauss-Jacobi quadrature rule appears to be incorrect. For $p=1$, the corresponding special case of (8) (derived originally in [8]) was obtained in [18] (but its convergence was considered only for analytic integrands $f(t))$ and, by different approaches, in [10]. A special case of this rule (for $p=1$ ) was originally derived in [12].

\footnotetext{
*There is an error in the formula in [10]. The corrected formula was supplied by the referee.
} 
It seems that, up to now, nowhere was the uniform (as opposed to pointwise) convergence of Gaussian quadrature rules for the numerical evaluation of (1) considered, even in the special cases $p=0$ and $p=1$. This will be done here on the basis of a classical theorem of convergence theory and the results of this section and [8]. Such a convergence is useful (particularly, for $p=0$ and $p=1$ ) in the numerical solution of singular integral equations appearing in several branches of physics and engineering.

It should be mentioned that the above Gaussian quadrature rules ((7) to (10)) should not be confused with the interpolatory quadrature rules based on Gaussian nodes (and sometimes called Gaussian rules too) suggested in [14], [19] for $p=0$. The convergence of the latter class of quadrature rules for $p=0$ was studied in detail both for pointwise [3]-[5], [9], [16], [21] and for uniform [14], [20], [21] convergence. From these results it follows that in certain interpolatory quadrature rules based on Gaussian nodes for the numerical evaluation of $I^{(0)}(x)$ for particular weight functions $w(t)$ uniform convergence does not hold (see, e.g., [21]).

Finally, for $x=a$ and $x=b, I^{(p)}(x)$ was defined by (2) (together with (6)) and, clearly, the corresponding Gaussian quadrature rule (7) remains valid. The present results for uniform convergence hold true along the whole interval $[a, b]$. Obviously, this does not mean that $\lim _{x \rightarrow c} I^{(p)}(x)$ exists or is equal to $I^{(p)}(c), c=a, b$, in general (but the integrals in the sum on the right side of (2) have evidently no influence on the error term $E_{n}$ ).

2. Proof of Convergence. By taking into account the previous results, we see directly that the error $E_{n}$ made when approximating (1) by the corresponding Gaussian quadrature rule (7) is equal to the error made when approximating the regular integral

$$
J^{(p)}(x)=\int_{a}^{b} w(t) g_{x}^{(p)}(t, x) d t, \quad w(t) \geqslant 0 \text { for } a \leqslant t \leqslant b,
$$

by the analogous quadrature rule for regular integrals, where [8]

$$
g_{x}^{(p)}(t, x)=\left\{\begin{array}{l}
\frac{p !}{(t-x)^{p+1}}\left[f(t)-\sum_{k=0}^{p} \frac{f^{(k)}(x)}{k !}(t-x)^{k}\right], \quad t \neq x \\
\frac{f^{(p+1)}(x)}{p+1}, \quad t=x
\end{array}\right.
$$

We assume that $f^{(q)}(x)$ is a Hölder-continuous function with index $\lambda, 0<\lambda \leqslant 1$, $\left(f^{(q)} \in H_{\lambda}\right)$ with $q \geqslant p+1$. Then, on the basis of a well-known theorem in approximation theory due to Gel'fond (see, e.g., [13]), there exists a sequence of polynomials $s_{n}(x)$ of degree not higher than $n$ such that

$$
\left|r_{n}^{(k)}(x)\right| \leqslant C_{1} n^{k-q-\lambda}, \quad k=0(1) q, a \leqslant x \leqslant b,
$$

where

$$
r_{n}(x)=f(x)-s_{n}(x)
$$

and $C_{1}$ denotes a positive constant. 
We wish to evaluate the error $E_{n}$ when approximating (1), or equivalently (11), by using the appropriate Gaussian quadrature rule. Clearly, this error remains unchanged if we apply the same rule to the evaluation of the integral

$$
K^{(p)}(x)=\int_{a}^{b} w(t)\left[g_{x}^{(p)}(t, x)-h_{n, x}^{(p)}(t, x)\right] d t,
$$

with $h_{n, x}^{(p)}(t, x)$ defined by a formula analogous to (12) with $f(x)$ replaced by $s_{n}(x)$, the polynomial of best uniform approximation to $f(x)$ and its first $q$ derivatives, (13). This is so because, obviously, $h_{n, x}^{(p)}(t, x)$ is a polynomial of degree $n-p-1$ (if $n \geqslant p+1$ ) or 0 (if $n<p+1$ ) and for such a polynomial the Gaussian quadrature rule with $n$ nodes is exact.

Now we take into account that

$$
\begin{aligned}
& g_{x}^{(p)}(t, x)-h_{n, x}^{(p)}(t, x) \\
& =\left\{\begin{array}{l}
\frac{p !}{(t-x)^{p+1}}\left[r_{n}(t)-\sum_{k=0}^{p} \frac{r_{n}^{(k)}(x)}{k !}(t-x)^{k}\right], \quad t \neq x, \\
\frac{r_{n}^{(p+1)}(x)}{p+1}, \quad t=x,
\end{array}\right.
\end{aligned}
$$

as is quite clear. Furthermore, since $r_{n}^{(q)} \in H_{\lambda}$ because the same was assumed for $f(x)$ and $s_{n}(x)$ is a polynomial, we conclude that

$$
g_{x}^{(p)}(t, x)-h_{n, x}^{(p)}(t, x)= \begin{cases}\frac{r_{n}^{(p+1)}(\xi)}{p+1}, & \xi \in(t, x), t \neq x, \\ \frac{r_{n}^{(p+1)}(x)}{p+1}, & t=x .\end{cases}
$$

Next, we use (13) with $k=p+1$ and we find

$$
\left|g_{x}^{(p)}(t, x)-h_{n, x}^{(p)}(t, x)\right| \leqslant \frac{C_{1}}{p+1} n^{p+1-q-\lambda}, \quad q \geqslant p+1
$$

Finally, by applying the Gaussian quadrature rule to $K^{(p)}(x),(15)$, and taking into account the previous developments, as well as the fact that

$$
\int_{a}^{b} w(t) d t=\sum_{i=1}^{n} \mu_{i, n}
$$

for the sum of the weights of this rule, we conclude directly that

$$
\left|E_{n}\right| \leqslant C_{2} n^{p+1-q-\lambda}, \quad q \geqslant p+1,
$$

where $C_{2}$ is a positive constant defined by

$$
C_{2}=\frac{C_{1}}{p+1} \int_{a}^{b} w(t) d t
$$

These results prove the uniform convergence of the Gaussian quadrature rule considered in [8] (and rederived by an alternative procedure here) and establish the 
corresponding rate of convergence. They can be stated in the form of the following theorem:

THEOREM. The n-point Gaussian quadrature rule for the numerical evaluation of Cauchy principal value integrals and their pth derivatives with a nonnegative generalized Hölder-continuous weight function along a finite integation interval converges uniformly in the closed integration interval for $n \rightarrow \infty$ for integrands $f$ such that $f^{(q)} \in H_{\lambda}(q \geqslant p+1)$, and the rate of convergence is $O\left(n^{p+1-q-\lambda}\right)$.

Concluding, we make three remarks: (i) As already mentioned in the introduction, a Cauchy principal value integral should be interpreted as a finite-part integral at the endpoints $a$ and $b$ of the integration interval. (ii) Since Gaussian quadrature rules for Cauchy principal value integrals are exact for polynomials of degree up to $2 n$ [5]-[7], [11], we could have used the sequence of polynomials $s_{2 n}(x)$ instead of the sequence of polynomials $s_{n}(x)$ in the previous developments; yet, the rate of convergence, (20), would not change although the values of the positive constants $C_{1}$ and $C_{2}$ in (13) and (21), respectively, would change. (iii) The previous results hold also for the Radau- and Lobatto-type Gaussian quadrature rules and, more generally, for convergent interpolatory quadrature rules for regular integrals when these rules are modified to apply to Cauchy principal value integrals and their derivatives [7], [8], [11]. Of course, in such a case we cannot use the sequence of polynomials $s_{2 n}(x)$ in place of the sequence of polynomials $s_{n}(x)$, although we may be able to use another appropriate sequence of polynomials $s_{m}(x)$ with $n<m<2 n$, the value of $m$ depending on the accuracy of the quadrature rule (e.g., $m=2 n-1$ for Radau-type rules and $m=2 n-2$ for Lobatto-type rules).

3. An Application. As an application, we consider the classical collocation method for the numerical solution of the dominant Cauchy-type singular integral equation of the first kind of the form

$$
\frac{1}{\pi} f_{-1}^{1}\left(1-t^{2}\right)^{-1 / 2} \frac{f(t)}{t-x} d t=g(x), \quad-1<x<1 .
$$

By applying the Gauss-Chebyshev method to the numerical solution of this equation, we obtain the following equations:

$$
\frac{1}{n} \sum_{i=1}^{n} \frac{f\left(t_{i, n}\right)}{t_{i, n}-x_{k, n}}+\frac{1}{\pi} E_{n}\left(f ; x_{k, n}\right)=g\left(x_{k, n}\right), \quad k=1(1)(n-1),
$$

where the nodes $t_{i, n}$ and the collocation points $x_{k, n}$ are the roots of the Chebyshev polynomials of the first kind $T_{n}(x)$ and of the second kind $U_{n-1}(x)$, respectively. We are interested in the error term $E_{n}$ (depending both on the integrand $f$ and on the node $x_{k, n}$ used). This application was also considered by Elliott [2].

By taking into account the theorem proved in the previous section, we conclude that if $f$ possesses a Hölder-continuous first derivative $\left(f^{\prime} \in H_{\lambda}\right)$, the quadrature rule used in (22) converges uniformly (for all $x_{k, n}$ ) as $n \rightarrow \infty$, that is,

$$
\left|E_{n}\left(f ; x_{k, n}\right)\right| \leqslant C_{3} n^{-\lambda}, \quad k=1(1)(n-1),
$$

( $C_{3}$ being a positive constant). It seems that this result cannot be improved. Nevertheless, Elliott proved in [2] a stronger convergence result, where the condition 
$f^{\prime} \in H_{\lambda}$ was replaced by the weaker condition $f \in H_{\lambda}$. The proof given in [2] has not been quite clear to the author, but the fact that Elliott dealt only with pointwise convergence and not with uniform convergence (as is the case here) may be the reason for the discrepancy between the results of Elliott and the present ones.

Here, we will establish a bound analogous to (24) by a second method, independent of the present results and based on the results of Sheshko [21], who proved that the error $E_{n}\left(f ; x_{k, n}\right)$ for the interpolatory quadrature rule based on the nodes $t_{i, n}$ (coinciding with the corresponding Gaussian quadrature rule for the collocation points $x_{k, n}$ and only for these points) is bounded by

$$
\left|E_{n}\left(f ; x_{k, n}\right)\right| \leqslant C_{4}\left(1-x_{k, n}^{2}\right)^{-1 / 2} n^{-\lambda} \log n, \quad k=1(1)(n-1),
$$

for $f \in H_{\lambda}$, whence

$$
\left|E_{n}\left(f ; x_{k, n}\right)\right| \leqslant C_{4}\left(1-x_{k, n}^{2}\right)^{-1 / 2} n^{-(\lambda+1)} \log n, \quad k=1(1)(n-1),
$$

for $f^{\prime} \in H_{\lambda}\left(C_{4}\right.$ denoting again a positive constant). Now we take into account the explicit formulae for the collocation points $x_{k, n}$ (roots of $U_{n-1}(x)$ )

$$
x_{k, n}=\cos (k \pi / n), \quad k=1(1)(n-1) .
$$

Then

$$
\left(1-x_{k, n}^{2}\right)^{-1 / 2}=1 / \sin (k \pi / n), \quad k=1(1)(n-1) .
$$

Therefore, for $n \rightarrow \infty$, we obtain from (26)

$$
\left|E_{n}\left(f ; x_{k, n}\right)\right| \leqslant C_{5} n^{-\lambda} \log n<C_{6} n^{-(\lambda-\varepsilon)}, \quad k=1(1)(n-1),
$$

$\left(C_{5,6}\right.$ being appropriate positive constants and $\varepsilon$ an arbitrarily small positive quantity). This result almost coincides with (24) (although obtained by quite a different method).

Acknowledgement. The results reported here belong to a research project supported by the National Hellenic Research Foundation. The financial support of this Foundation is gratefully acknowledged.

Chair of Mathematics B'

School of Engineering

University of Patras

P.O. Box 1120

GR-261.10 Patras, Greece

1. P. J. Davis \& P. Rabinowitz, Methods of Numerical Integration, 1st ed., Academic Press, New York, 1975.

2. D. Elliotr, "On the convergence of Hunter's quadrature rule for Cauchy principal value integrals," $B I T$, v. 19,1979 , pp. $457-462$.

3. D. Elliotr \& D. F. PAGET, "On the convergence of a quadrature rule for evaluating certain Cauchy principal value integrals," Numer. Math., v. 23, 1975, pp. 311-319.

4. D. Elliott \& D. F. PAGET, "On the convergence of a quadrature rule for evaluating certain Cauchy principal value integrals: an addendum," Numer. Math., v. 25, 1976, pp. 287-289.

5. D. Elliotr \& D. F. PAGET, "Gauss type quadrature rules for Cauchy principal value integrals," Math. Comp., v. 33, 1979, pp.301-309.

6. D. B. HUNTER, "Some Gauss-type formulae for the evaluation of Cauchy principal values of integrals," Numer. Math., v. 19, 1972, pp. 419-424.

7. N. I. IoAkimidis, General Methods for the Solution of Crack Problems in the Theory of Plane Elasticity, Doctoral Thesis at the National Technical University of Athens, Athens, 1976. [Available from University Microfilms, Ann Arbor, Michigan; order no. 76-21,056.] (Greek) 
8. N. I. IOAKIMIDIS, “On the numerical evaluation of derivatives of Cauchy principal value integrals," Computing, v. 27, 1981, pp. 81-88.

9. N. I. IOAKIMIDIS, "Further convergence results for two quadrature rules for Cauchy type principal value integrals," Apl. Mat., v. 27, 1982, pp. 457-466.

10. N. I. IOAKIMIDIS, “On the numerical evaluation of a class of finite-part integrals," Z. Angew. Math. Mech., v. 63, 1983, pp. 572-574.

11. N. I. IOAKImidis \& P. S. Theocaris, "On the numerical evaluation of Cauchy principal value integrals,” Rev. Roumaine Sci. Tech. Sér. Méc. Appl., v. 22, 1977, pp. 803-818.

12. N. I. IOAKImidis \& P. S. Theocaris, "On the numerical solution of singular integrodifferential equations," Quart. Appl. Math., v. 37, 1979, pp. 325-331.

13. A. I. KalandiYA, Mathematical Methods of Two-Dimensional Elasticity, 1st English ed., p. 108, "Mir", Moscow, 1975.

14. A. A. KorneICHUK, "Quadrature formulae for singular integrals," Zh. Vychisl. Mat. i Mat. Fiz., v. 4, no. 4, suppl., 1964, pp. 64-74. (Russian)

15. H. R. KUTT, On the Numerical Evaluation of Finite-Part Integrals Involving an Algebraic Singularity, CSIR Special Report WISK 179, National Research Institute for Mathematical Sciences, P.O. Box 395, Pretoria 0001, South Africa. [Available also from NTIS (National Technical Information Services), U.S.A.]

16. G. Monegato, "The numerical evaluation of one-dimensional Cauchy priricipal value integrals," Computing, v. 29, 1982, pp. 337-354.

17. N. I. MuSKhelishvili, Singular Integral Equations, 2nd English ed., p. 228, Wolters-Noordhoff, Groningen, 1958.

18. D. F. PAGet, “The numerical evaluation of Hadamard finite-part integrals," Numer. Math., v. 36, 1981, pp. 447-453.

19. D. F. PAGet \& D. ElliotT, “An algorithm for the numerical evaluation of certain Cauchy principal value integrals," Numer. Math., v. 19, 1972, pp. 373-385.

20. D. G. SANIKIDZE, “On a uniform estimation of approximation of singular integrals with Chebyshev's weighted function by sums of interpolating type," Soobshch. Akad. Nauk Gruzin. SSR, v. 75, no. 1, 1974, pp. 53-55. (Russian)

21. M. A. SHESHKo, “On the convergence of quadrature processes for a singular integral," Soviet Math. (Iz. VUZ), v. 20, no. 12, 1976, pp. 86-94. (English)

22. G. J. TSAmasphyros \& P. S. Theocaris, "On the convergence of a Gauss quadrature rule for evaluation of Cauchy type singular integrals," BIT, v. 17, 1977, pp. 458-464. 